- Dental practitioners within Phase 1 Personal Dental Services Pilot schemes felt that their participation was valuable and positive.

Practitioners perceive that the PDS pilot scheme allowed them to improve the quality of care they delivered to patients and the standard of management within the practice.

- Benchmarking performance and ensuring that mechanisms for monitoring and improving the quality of service were felt to be important components to the development of new systems of remuneration, but had been largely overlooked in the pilot schemes.

- Local commissioning should identify mechanisms for ensuring the effective planning, management and evaluation of changes in remuneration.

\title{
A qualitative analysis of the planning, implementation and management of a PDS scheme: Lessons for local commissioning of dental services
}

\author{
J. T. Newton, ${ }^{1}$ B. Alexandrou, ${ }^{2}$ B. Dixon Bate ${ }^{3}$ and H. Best ${ }^{4}$
}

Aim To identify the experiences of primary care trust employees, the dental teams and other key individuals of the planning, implementation and management of a Personal Dental Services scheme.

Method A thematic analysis of a series of qualitative interviews with 29 individuals who were involved in the planning, implementation and management of a PDS scheme in South East London.

Findings Nine key themes were analysed from the data. For each theme perspectives could be identified for both the employees of the primary care trusts and the dental team. These perspectives differ in key respects. Conclusions Practitioners value the PDS scheme and consider it a positive experience. They suggest that it has led to an increase in quality of care, and a more professional management approach to the practice. The practice team felt that they have benefited from an enhanced working environment. The main concern expressed was that patient registrations were not being accurately assessed. Those involved in the management of the PDS scheme, while endorsing local commissioning arrangements, were concerned that it was not known whether PDS was meeting local needs. There was little quality benchmarking, which would have allowed robust measure of success. The contract model and outcomes should have been more sensitively designed. There was concern expressed that the small number of practices who participated in the pilot scheme prohibits the possibility of thoroughly analysing the impact of local commissioning. Future local commissioning should identify mechanisms for ensuring the effective planning, management and evaluation of the impact of the schemes. A core element of this will be the specification of appropriate goals for commissioning.
1"Professor of Psychology as Applied to Dentistry, GKT Dental Institute: 2,3 Independent Research Consultants, London; ${ }^{4}$ Specialist in Dental Public Health, GKT Dental Institute *Correspondence to: Professor Tim Newton, GKT Dental Institute, Caldecot Road, London, SE5 9RW

Email: tim.newton@kcl.ac.uk

\section{Refereed paper}

Accepted 28 June 2005

DOI: $10.1038 /$ sj.bdj.4813643

( $)$ British Dental Journal 2006; 200: 625-630

\section{BACKGROUND}

Changes to the funding and commissioning of dental services in England within the terms of the Health and Social Care Act mean that dental services can be designed locally to meet local needs. ${ }^{1,2}$ Within a general framework of commissioning dental services, Primary Care Trusts have greater flexibility in contracting arrangements to ensure that services meet the needs of the localities served by each PCT. Many of the changes outlined in NHS dentistry: Options for change, ${ }^{3}$ and subsequently enshrined in the Health and Social Care Act, were initially tested in the Personal Dental Services pilot schemes, and it has been proposed that the experiences of the PDS schemes should inform the implementation of local commissioning. ${ }^{4}$ The aim of the present study is to identify the experiences of primary care trust employees, certain other key personnel and the dental teams involved of the planning, implementation and management of a Personal Dental Services Scheme.

Personal Dental Services were conceived as an alternative method of remunerating general dental practitioners in part to allow local commissioning of general dental services as a response to perceived problems of gaining access to NHS dental care. The Bloomfield Report, ${ }^{5}$ the Health Select Committee report and the Department of Health document Improving NHS dentistry ${ }^{6}$ all suggested that alternatives should be sought to the only system then existing for payment of dental practitioners in general dental practice, that is, a national contract and a nationally negotiated scale of fees.

The Bloomfield report was central to the development of Personal Dental Services. It identified several key points:

- NHS dental services should be needs led rather than demand led.

- Services should be locally managed with resources allocated accordingly

- A style of service involving greater prevention and support for individual self maintenance of oral health was also identified as being relevant to the health status of the population at this period of time.

But the report indicated that the profession had not developed a consensus for further health policy and health system develop- 
ments. For remuneration systems, there was widespread dissatisfaction among the profession with the current system and the two main themes that emerged involved the perpetual 'treadmill effect' and the current system of payment did not reward quality dental care but the quantity of care. The concept of an average practitioner doing an average amount of work was no longer viable nationally. It was identified as being flawed in terms of geographic variations in costs and individual commitments of time and workload to the NHS. ${ }^{5,7}$

Following a period of consultation between the Department of Health and representatives of the dental profession, legislation was passed which allowed for the implementation of local commissioning in the form of the NHS (Primary Care) Act of 1997. This act, together with the white paper Choice and opportunity ${ }^{8}$ outlined a scheme whereby pilot projects would investigate the impact of local commissioning on provision of NHS dental care. Initially 15 proposals for different PDS schemes were approved nationally in the 'first wave' of schemes.

A national evaluation of Personal Dental Services schemes was commissioned by the Department of Health as part of its Policy Research Programme. The contract for this evaluation was won following a process of competitive tender by a team of researchers based at the University of Birmingham. A combination of quantitative and qualitative approaches were employed by the University of Birmingham team focussing on both agreed performance measures and the experiences of the dental teams involved in the PDS schemes, and the commissioners of the schemes. The specific research methods employed by the University of Birmingham team were:

- An analysis of key documentation associated with PDS schemes, for example contracts, business and reports.

- Fieldwork visits to 20 PDS sites, including:

- Face-to-face interviews with staff

- Annual collection of quantitative data for each site, related to the objectives of each PDS scheme

- A postal survey of all members of the dental team

- Telephone interviews with representatives from Local Dental Committees in the areas of the PDS schemes

- Telephone interviews of health authority leads for PDS schemes

- Postal survey and interviews with users of the service (patients).

The national evaluation was published in July $2003^{9}$ and summarised in a series of key articles. ${ }^{4,10-12}$ There were several findings of relevance to the implementation and management of the schemes. These can be summarised as follows:

- Organisation and management of PDS schemes was considered to be time consuming, and health authorities played a key role in this process

- All pilot PDS schemes assessed their progress as the same as or better than expected

- Pilot PDS schemes largely considered that they had achieved their goals

- Local contracting was perceived as more sensitive and responsive to the needs of the local population

- Capitation based pilots identified their strengths as increasing the retention of participating dentists within the NHS and improved availability of primary dental care for the local population

- Information Technology problems and difficulties in communication with the Dental Practice Board were identified as a problem with the initial implementation of the scheme. This emerged as a problem in obtaining reliable and timely information on the schemes

- Lack of funding for growth and the uncertainty of the future for PDS schemes undermined the ability of practices to plan ahead and develop

- Providers in PDS were required to develop management skills

- PDS dentist perceived that there were great advantages to removing the drive to ensure large numbers of treatments were carried out in order to ensure income, as necessitated on fee-per-item remuneration systems

- PDS was seen as improving access to NHS dentistry

- There was little use of professionals complementary to dentistry in the pilot PDS schemes

- Patients were generally unaware of the change to PDS and had little knowledge of PDS.

Personal Dental Services (PDS) was established as a Pilot Project in October 1998 within the Lambeth, Southwark and Lewisham (LSL) Health Authority and was one of the first wave PDS schemes. Entry to PDS was open to all local dental practitioners who met certain key criteria produced by the Health Authority. Seven practices joined the scheme and at the time of this study there were five practices remaining with over 20 dentists involved. The programme involved new ways of contracting with providers, closer working between PDS and Community Dental Services, skill mix and innovative methods of promoting uptake of services. The scheme is now under the auspices of Southwark Primary Care Trust.

The specific objectives for Personal Dental Services within Lambeth, Southwark and Lewisham were as follows:

- To improve the uptake of dental services among local residents, particularly children with high caries rates

- To deliver dental care of appropriate quality

- To ensure appropriate, quality safety net and specialist services are available for all residents of the area.

\section{METHOD}

As part of a larger evaluation of PDS within the three Primary Care Trusts of Lambeth, Southwark and Lewisham, and in order to inform local commissioning of dental services, an analysis of the implementation and management of the Personal Dental Services scheme in these areas was undertaken. Using qualitative interview methods this study sought to identify the experiences of primary care trust employees, and the dental teams of the planning, implementation and management of a Personal Dental Service, in addition certain key individuals who had been involved in the setting up of the scheme but who were no longer working either in the practices or the PCT were also approached. All the field work for the study was undertaken by two of the authors (BA and BDB); both are experienced in these methods. Interviews were largely unstructured following the recommendations of Britten. ${ }^{13}$ All interviews were tape recorded and transcribed for analysis.

A total of 29 interviews were conducted. The participants included roughly equal numbers of individuals based within the Commissioning body and in the PDS practices. The sample was derived by purposive sampling. The research team identified key individuals involved in the planning, implementation and management of the schemes. There were two individuals who declined to participate - the reasons given were that they no longer worked in the area or in connection with the PDS scheme and thus felt that their input could not be relevant to the project. The sample comprised individuals from a range of backgrounds in both the commissioning body and the practices. Within the commissioning body, representatives from finance and management, public health and primary care were included; within the practices, individuals from all the professional groupings were included.

The data were analysed by thematic analysis within the framework of grounded theory. ${ }^{14}$ Analysis was conducted by 
two researchers. The researchers sought to describe consensus in views; though where differences of view were found these were noted and analysed.

\section{FINDINGS}

The findings from the in-depth interviews were summarised as a series of nine themes. These themes were common to both the management team within the Primary Care Trusts, and the dental team working in the PDS practices. However the perspectives that each of these groups took to the individual themes differed. The themes identified in the interviews were as follows:

- PDS development and objectives

- Patient registration - number and type

- Quality of care

- Dental practice skill mix

- Health promotion

- Financial reconciliation

- Dental practice management

- Performance monitoring

- Future developments.

Each theme will be explored separately. For each theme the views of the two groups will be summarised. Please note that the themes summarise the perceptions of the participants and may not be supported by objective data. As such they provide an insight into biases and heuristics used by the participants in the decision making process.

\section{PDS development and objectives}

The management perspective

The management perspective viewed PDS within a broad government framework to contain expenditure on dental fees which were perceived as burgeoning within the GDS. Concurrent with this they perceived a general shift (and in 2005 a wholesale shift) to local commissioning of dental services by PCTs. The Department of Health encouraged PCTs to pilot local commissioning arrangements through the PDS scheme. Within Lambeth, Southwark and Lewisham the general dental service was felt to not specifically promote quality of care - as well as producing large variations in quality, and that therefore alternative mechanisms of funding were felt to be necessary to take dentists off the 'treadmill' and better manage PCT resources.

There was broad agreement within the management side that the key objectives of PDS were to:

- Meet local need - and in particular increase uptake of dental services by children (Lambeth, Southwark and Lewisham were identified as having low registration rates for children)

- Improve quality of care:

through providing alternative incentives to dentists

'moving away from volume of work to undertake preventative work' [PCT interviewee]

- Promote greater integration of services and targeting specialist services where most required

- Provide financial stability for dentists to enable them to be more prospective in their planning.

'We had three main objectives of the pilot-one was to increase the uptake of children - one was to help practitioners improve the quality of their care - and the third one - was trying to integrate different aspects of dental services under the same roof' [PCT interviewee].

There was a perception that the last objective (providing financial stability) had tended to dominate the contracting process.

Those involved in the management and implementation of the PDS scheme raised a number of issues with this approach. First, the timing imposed on the development of the PDS scheme by the Department of Health may have resulted in 'too much too soon'.
Specifically, dental practices may have been overwhelmed by the required changes, and Information Technology systems could have had greater time to develop - particularly in aligning with DPB requirements - as well as perhaps being better resourced. Overall it was felt that phasing in the scheme may have been more desirable. The employees also emphasised that at the time of setting up the PDS pilot scheme there was little experience of capitation schemes, for the staff to draw upon, and that in terms of useful lessons for the future it is possible that there were too few practices in the pilot schemes to allow generalisations to be made.

There was a general feeling that the contracting model was too 'crude' or 'simple' - possibly as a result of 'conflicting objectives' and potentially a 'too' inclusive approach. Related to this there were questions raised by the PCT employees over whether financial reconciliation was too great a priority for some practitioners in developing the model, and whether setting budgets on the historic income of practices could be considered accurate.

A key criticism for the employees of the PCTs was that the model failed to build in any real performance monitoring. The feeling was expressed that quality standards were not properly articulated and incorporated into the model. The model provides an incentive to increase patient numbers but does not necessarily ensure appropriate treatment and advice. Some employees commented that while there had been attempts to build in monitoring and standards, these had not been fully developed.

Finally the PCT employees questioned whether the capitation model could become another form of the 'treadmill' as it may result in practitioners simply focusing on registration numbers.

\section{The dental practitioners' perspective}

The general dental service fee-per-item system for adults was perceived as flawed and encouraged speed rather than quality. The practitioners were looking for a system that would remove them from the stress of the treadmill, and allow them to focus on patients' needs, incorporating a greater preventive approach. Practitioners also identified the need to increase the uptake of dental services among children in the area. Finally it was identified that PDS gave the opportunity to have a stable financial base from which to plan practice development.

Some dentists raised what they perceive as a fundamental issue in increasing patient numbers:

'at the end of the day if we take on more patients to try and keep our numbers up and all of those patients need ten times more treatment than the patients needed last year, yes we will have lots of patients registered but we won't have the time to do their treatment... so it is a bit of a vicious cycle' [Associate Dentist].

The dental practice team raised three specific issues concerning the implementation of the PDS scheme. First the development of business plans and other set up requirements were onerous for some. Second, there were a number of Information Technology problems in the setting up of the scheme, and incompatibility between the IT system implemented and the Dental Practice Board system.

\section{Patient registration - number and type} The management perspective

The central issue surrounding patient registration for those involved in the management of the PDS scheme within the three Primary Care Trusts was whether there had been an increase in registrations, and by inference whether PDS had reached one of its primary objectives. The increase in the registration period clouded the issue of whether there has been a genuine increase in patient numbers attributable to the PDS, since those who would have originally fallen out of the system remain for a further nine months. There was perception from some interviewees that recall of old patients had been used to increase numbers - rather than recruiting those historically not in the system. The inability to 
monitor change in registration was further compounded since the initial information on patient registrations from the DPB was compromised by flaws in the IT system and failure of dental practitioners to submit appropriate forms.

The issue of the mix of NHS and private care under PDS was also discussed. There was some feeling within the PCT employees that this was now different to pre-PDS.

As a consequence of these concerns, many of the PCT employees did not feel able to confidently surmise whether 'local need' has been met. Some believe that more children have been registered (but by virtue of CDS provision within practices), others interviewed felt that patient numbers have actually reduced. One simply had 'no idea on this'. Finally there was a minority view that whatever approach is taken there will always be a proportion of the population who simply will not visit a dentist unless in trouble - thus raising the question of whether registration of the whole population is an appropriate goal.

\section{The dental practitioners' perspective}

All practices believe their patient numbers have increased. Furthermore, some feel there is a disparity between DPB figures 'if they knew what they were doing there would be more' [Principal Dentist]. One practice believed it had 7,000 more patients registered than was 'officially' recognised. One practice employee felt that PDS had enabled them to see refugees and more patients on income support. The key mechanism of the increase in registrations is thought by practitioners to be through word of mouth:

'I definitely have more and more kids coming in on recommendations' [Associate Dentist].

One practice's administrative staff indicated that all patients that had fallen off the practice list were re-contacted to register, thus supporting the concerns expressed by the management interviewees.

\section{Quality of care}

\section{The management perspective}

The management interviewees felt overwhelmingly unable to say whether treatment patterns or quality of care has changed under PDS. This was perceived to be due to inadequate performance monitoring. Information on treatments performed and the quality of these varies - and depends on ' what they tell the PCT' [PCT employee]. One commented that 'a more thorough review of treatment patterns is necessary'.

\section{The dental practitioners' perspective}

There was absolute consensus among the dental team that dentists are able to spend more time on patients under PDS, particularly in providing advice and information on maintaining oral health:

'You get paid for just talking to patients and telling them what is going on in their mouth' [Associate Dentist].

The standard of treatment was felt to be higher as a consequence of not being constrained by time. There is also a general feeling that treatment is more appropriate to patient needs - although some argued that this also occurs under the terms of the GDS. Many associates and vocational dental practitioners highlight how the PDS enables them to work in the manner in which they were trained, that is focussing on quality, not on speed and volume of treatment.

However, one practitioner expressed the view that the 'average' patient is better off under PDS but that those with high treatment needs were unlikely to improve under PDS.

\section{Dental practice skill mix}

\section{The management perspective}

There was general consensus among the PCT employees that skill mix was the responsibility of the dental practice. Little guid- ance was given by the PCT on the employment of professionals complementary to dentistry, though it was hoped that increased skill mix would result from greater business planning. In terms of management, all practices were thought to have practice managers following the introduction of PDS. There is a general feeling that skill mix has increased although not as much as the opportunity allowed - and questions were raised as to whether the PDS funding mechanism promoted it. The key addition to practice services was thought to be the introduction of CDS practitioners into the PDS practices under Service Level Agreements. However the strong conviction was expressed that they should 'never be in the inner set-up of practices' [CDS manager].

\section{The dental practitioners' perspective}

The development of the practice manager role was highlighted by most practices. The introduction of CDS dentists for the treatment of children with high needs was regarded as a beneficial outcome of the PDS and had the secondary benefit of other family members joining practice. One practice, which was unable to house a CDS specialist, believed that the greater time available for treatment under PDS has meant that there is less requirement for one. PDS had little consequence on the employment of hygienists. In addition practices pointed to the development of an oral health educator role as an example of skill mix development.

\section{Health promotion}

\section{The management perspective}

The 'management' perspective on health promotion related primarily to the Community Dental Service. There was a feeling that health promotion should have been built into the practices' working style and should operate by sending individuals out of the practice, but that in contrast the practices wanted the health promotion to be 'bolted on' to existing service provision. Consequently CDS efforts to train existing dental practice staff were not felt to have been successful. PDS was not felt to be a catalyst for dentists to undertake health promotion outside the practice in the wider community.

\section{The dental practitioners' perspective}

The key health promotion benefits of PDS for the dental team are thought to come from the greater time spent on giving patients advice. There was some evidence of specific health promotion activities: two practices had engaged oral health educators, working with patients referred by the dentist, one practice had trained a nurse as an educator - although time constraints made this role very limited. There were various levels of oral health educational materials in practices. One practice has taken the initiative to visit local pre-schools to 'make visiting the dentist fun'.

\section{Dental practice management \\ The management perspective}

This issue was not discussed by the 'management' interviewees.

\section{The dental practitioners' perspective}

There was general consensus among the dental practice members that they are able to plan improvements to practice, staff and services better. For all practices the distinct benefit of working in PDS was perceived as improved staff morale among both clinicians and administrative staff:

'The dentists used to be under so much pressure and ended up running late, it's a lot smoother now and better for everybody. I've worked for the surgery for 36 years and think this is fantastic' [Member of administrative staff of practice].

There was a perception that PDS encouraged greater 'open management' in the practices, for example staff meetings in some 
practices. Training and development was felt to be a much higher priority than before, and two practices had Investing In People awards, with one about to be awarded.

The impact of PDS funding on staff turnover not clear, for some practices this was never an issue, for others it was and remains so. Administration staff highlighted the 'lack of red tape' and no requirement for prior approval as key benefits for practice and patients. Opening hours do not appear to have been affected.

\section{Financial reconciliation}

The management perspective

The key issue here was a large fall in patient charges which had occurred during the course of the PDS scheme.

\section{The dental practitioners' perspective}

For the dental practices financial stability was the key success of PDS. There was some recognition that under PDS earning potential is capped - unlike under GDS - therefore it was thought that PDS was likely to attract a certain type of dentist.

\section{Performance monitoring}

The management perspective

There was a general feeling that quality standards were not properly specified: 'The outcomes were set in a woolly manner' [PCT employee]. Furthermore the contract did not incorporate adequate performance monitoring mechanisms: 'The existing system is not performance sensitive' [PCT employee].

Information from the DPB was available on patient registration and treatment activity, but it was generally felt that such activity data did not allow the PCT to understand whether quality has actually improved: 'DPB data was all historical, doesn't tell you what you need to do, tells you what you have done' [PCT employee].

There were also questions raised as to whether the PCT had sufficient expertise to interpret any of the data. It was felt that there was a missed opportunity to conduct oral health assessments to provide a benchmark on quality that could be subsequently measured.

\section{The dental practitioners' perspective}

There was little comment from practitioners regarding performance monitoring. Some practitioners felt that performance monitoring was inadequate and focused solely on patient registrations. One practice concurred with management in the need for conducting an initial oral health assessment, and another believed that they set and meet their own quality standard targets.

\section{Future developments}

\section{The management perspective}

Local commissioning was felt to be the right approach as it fosters closer links between PCT and dental practitioners which can be built upon. It was seen as imperative that PCTs gain a greater appreciation of the time and resources required to develop local contracts, and a greater understanding of target groups and thier needs must underpin any future arrangements. There should be absolute clarity on outcomes and information requirements should be built into any system, for example building contractual obligtions to encourage preventative work. The desire to have a universal method of contracting with practices should be resisted - since it was perceived that simple models don't always work - rather, specific areas within the PCT area should be given specific models. Given that practices that joined the original PDS were highly selected group there was a debate over whether the existing culture amongst wider dental profession is compatible with the PDS approach. Finally it was perceived that there is a broader challenge to bringing dental services within the framework of all health services.

\section{The dental practitioners' perspective}

Those practices currently working within PDS had a narrower development agenda, which focused primarily on how the practices will develop in the future. Unease was expressed that the scheme will change wholesale. The practitioners hoped that dialogue and support from the PCT would continue. It was recognised that there is an opportunity to develop better auditing arrangements.

\section{DISCUSSION AND CONCLUSIONS}

The primary purpose of the research described here was to identify the experiences of primary care trust employees, and dental teams of the planning, implementation and management of a Personal Dental Services Scheme. Related to this it is hoped that this research will identify important principles which could be taken forward into the future local commissioning of dental services. It is important to note that the perspective of practitioners and that of PCT employees differed on many key points, and also in priorities. A particular challenge for local commissioning will be drawing together these disparate views. Furthermore, the perceptions of both sides may not be supported by objective evidence. For example it is interesting to note that the perception of those in the commissioning body was that PDS was designed to control expenditure and ensure quality, there is little evidence to suggest that either of these was an objective problem. Such beliefs, while not supported by evidence, are likely to consciously or sub-consciously influence the process of contract negotiation.

The PDS scheme sought to support practitioners to deliver appropriate quality dental care. From the in-depth interviews conducted with the primary care trust staff involved in the planning, implementation and management of the PDS scheme, it is apparent that they feel there is simply insufficient information available to judge the quality of dental care provided. On a positive note the dental practices strongly endorse the view that PDS has allowed them to deliver a higher quality of care, particularly preventive care.

It had been hoped that the introduction of PDS would allow practitioners to implement a greater emphasis on preventive interventions. The practitioners believe that this happened and was largely related to the additional time practitioners felt that they could spend with patients. Health education was viewed as taking place within the practice, though there was limited outreach of health promotion activities.

The availability of closer links to the Community Dental Service was perceived to create greater opportunity for the treatment of children and particularly those who were anxious about visiting the dentist, though the size of this effect was not quantified. However, interview participants identified that this was not a true integration of services, but the placing of outreach facilities into primary care.

Lessons are also identified for the future development of dental services. These are detailed below, but mirror the concerns identified in the Birmingham national evaluation of PDS schemes. ${ }^{4,10-12}$ These primarily concern the performance and quality management of the contracts.

The aims and objectives of any new scheme for commissioning dental health services should be clearly stated, in terms which are amenable to external verification. Key indicators of performance should be identified and monitored prior to the introduction of the new scheme. These indicators should include a range of indices reflecting all aspects of the evaluation of health services as suggested by the Department of Health. ${ }^{15}$ Specifically:

1. Assessment of fairness of provision relative to need

2. Assessment of efficiency and value for money

3. Assessment of effectiveness 
4. Evaluation of responsiveness to patient preferences

5. Evaluation of integration across professional inputs

6. Perceived flexibility in organisational forms and arrangements for staff

7. Local accountability.

Consideration should be given to the inclusion of indices of oral health related quality of life as outcome measures for health services. ${ }^{16,17}$ Current indices concentrate on indicators of disease, past disease experience and treatments performed. Systems for the capture, and analysis of information relevant to the evaluation of services need to be developed.

The present study adds to the national evaluation of PDS by providing an in-depth analysis of the views of individuals both in the commissioning body and the practices. It is interesting to note that discrepancies exist between these two sets of views. For example in the area of Health Promotion, the commissioning body appears to be looking for developments outside the practice which support the health of the community, whereas the practices view health promotion as comprising individual health education advice and to a latter extent activities directed at encouraging greater attendance at the practice. Perhaps crucial to these differences in perspective is the fundamental contrast in the aims of the scheme for the two sets of respondents. This pilot PDS scheme demonstrates how implementation of a new system against a tight deadline can lead to a focus on setting up the system to the possible neglect of consideration of the goals of the scheme, its monitoring and implementation.

The research described here was funded by Southwark Primary Care Trust. The evaluation received ethical approval from the King's College Hospital Research Ethics Committee and research governance approval from Southwark Primary
Care Trust. The authors would also like to thank the participants for their assistance with the research, their active participation and for facilitating access for the research team.

1. The Health and Social Care (Community Health and Standards) Bill. London: HMSO 2003.

2. Watson M. The Health and Social Care Bill. Br Dent J 2003; 195: 637-638.

3. NHS dentistry: Options for change. London: Department of Health, 2002

4. Goodwin N, Morris A J M, Hill K B et al. National evaluation of personal dental services (PDS) pilots: Main findings and policy implications. Br Dent J 2003; 195: 640-643.

5. Bloomfield K. Fundamental review of dental remuneration. Report of Sir Kenneth Broomfield KCB. London, December 1992.

6. Department of Health. Improving NHS dentistry. London: Department of Health 1994.

7. Palmer J. Bloomfield and ... what now? Comm Dent Health 1994; 11: 63-65.

8. Department of Health. Choice and opportunity (Primary care: the future). London: HMSO, 1993.

9. Hill K, Goodwin N, Bradnock G et al. National evaluation of personal dental services: Interim report to the Department of Health. University of Birmingham: School of Dentistry and Health Services Management Centre, 2001.

10. McLeod H S T, Morris A J. Evaluation of personal dental services (PDS) first wave pilots: The alternative to General Dental Services (GDS) offered by the capitationbased pilots. Br Dent J 2003; 195: 644-650.

11. Hall A C, Hill K B, Goodwin N et al. National evaluation of personal dental services: The perspective of dentists and professionals complementary to dentistry. Br Dent J 2003; 195: 651-653.

12. Hill K B, Hall A C, Morris A J et al. National evaluation of personal dental services: A qualitative investigation into patients' perceptions of dental services. Br Dent J 2003; 195: 654-656.

13. Britten N. Qualitative interviews in medical research. Br Med J 1995; 311: 251 253.

14. Richie J, Spencer L. Qualitative data analysis for applied policy research. In Bryman A, Burgess R (Ed) Analyzing qualitative data London: Routledge, 1994

15. Department of Health. Quality and performance in the NHS: Performance assessment framework. London: Department of Health, 1999.

16. Slade G D (Ed.) Measuring oral health and quality of life. Chapel Hill: University of North Carolina Dental Ecology, 1997.

17. Burke F J T, Wilson N H. Measuring oral health: an historical view and details of a contemporary oral health index (OHX). Int Dent J 1995; 45: 358-370. 